\section{Patientenratgeber Kopfschmerzen und Migräne}

Charly Gaul et al. Patientenratgeber Kopfschmerzen und Migräne. Berlin: ABW Wissenschaftsverlag 2020, 4. Auflage, 172 Seiten, 40 Abbildungen, 17,95 Euro, ISBN 9783940615619

Es gibt eine Fülle von Patientenratgebern bei Kopfschmerzen und Migräne. Aus diesen ragt das vorgelegte Buch hervor, indem es umfangreich und in wissenschaftlich fundierter Weise über Kopfschmerzerkrankungen berät und zur Eigentherapie anregt. Der Erfolg dieses Patientenratgebers drückt sich darin aus, dass er jetzt in der 4. Auflage vorliegt. Gegenüber der letzten Auflage hat sich insbesondere im pharmakologischen Bereich einiges getan, sodass neue Medikamentengruppen aufgenommen werden und andere neu eingeordnet werden mussten. Weiterhin sind Stimulationsverfahren in der Therapie etabliert worden, die ebenfalls neu besprochen worden sind. Außerdem haben sich die Autoren geändert, sodass jetzt vor allem die Erfahrungen der Migräneklinik in Königstein in das Buch eingegangen sind. Auch die moderne elektronische Patientenberatung findet Eingang in dieses Buch.

Der Vorteil dieses Buches liegt in seiner guten Handhabbarkeit. Es ist ausschließlich für Betroffene gedacht und geschrieben, dies wirkt sich positiv aus. Bei anderen Ratgebern ist gelegentlich die Tendenz zu beobachten, dass sie auch Lehrbuchcharakter haben möchten. Positiv sind die zahlreichen Beispiele, wie sich Betroffene selber helfen können und wann sie ärztliche Hilfe in Anspruch nehmen sollten. Sicherlich wird auch diese Auflage weite Verbreitung finden.

Stefan Evers, Coppenbrügge

\section{Persönlichkeitsstörungen}

Babette Renneberg, Sabine C. Herpertz. Persönlichkeitsstörungen, Reihe: Fortschritte der Psychotherapie Band 79. Göttingen:

Hogrefe Verlag 2021, 120 Seiten, 19,95 Euro, ISBN 9783801725082

Ein Buch, das zur rechten Zeit kommt! Mit dem für 2022 erwarteten ICD-11 steht im Bereich der Persönlichkeitsstörungen ein Paradigmenwechsel an. Das kategoreale Diagnosesystem weicht einem dimensionalen Ansatz, was nicht nur für die Diagnosestellung, sondern auch für das Verständnis und die Psychotherapie bedeutende Konsequenzen haben dürfte. 2 ausgewiesene Expertinnen, Babette Renneberg und Sabine Herpertz, gelingt es, prägnant darzustellen, auf was es bei der Diagnostik und Therapie der Persönlichkeitsstörungen in Zukunft ankommen wird. Beiden Autorinnen stellen die Systematik des neuen Diagnosekatalogs für die Persönlichkeitsstörungen in einer leicht nachvollziehbaren Weise vor. Aus der klinischen Perspektive kommen sowohl die potenziellen Vorteile als auch Schwierigkeiten der Neuerungen zur Sprache. In einem jeweils störungsbezogenen Kapitel werden die herkömmlichen Persönlichkeitsstörungskategorien in das Modell der dimensionalen, prominenten Persönlichkeitsmerkmale „übersetzt“. Diese Vorgehensweise macht es für den Kliniker leichter, sich mit dem neuen System zurechtzufinden. Die Darstellung der einzelnen Persönlichkeitsstörungen beinhaltet neben einer griffigen, auch in der Kommunikation mit dem Patienten leicht annehmbaren Beschreibung der problematischen Persönlichkeitsmerkmale eine Fallvignette, ein behaviorales Störungsmodell sowie eine kurze Übersicht zu Wirksamkeitsstudien. Besonders im Fokus stehen aber die Empfehlungen zum verhaltens- therapeutischen Herangehen. Hierbei ist es gelungen, trotz der Kompaktheit des Buches neben praxisnahen Interventionen Beispieldialoge und Rollenspiele unterzubringen.

Renneberg und Herpertz geht es um einen pragmatischen, verständnisorientierten Zugang zu Menschen mit Persönlichkeitsstörungen. Durch ihre Art der Aufbereitung wird der neue, in Dimensionen denkende Ansatz deutlich, der sich von der starren Zuordnung mehr oder weniger arbiträren Persönlichkeitskategorien befreien will. Aus diesem Blickwinkel ist es auch nicht weiter störend, dass die Autorinnen die schizotype und die schizoide Persönlichkeitsstörung anhand eines Patientenbeispiels, auf das beide Merkmale zutreffen, gemeinsam in einem Kapitel bearbeiten.

Ein kleiner Wermutstropfen ist, dass die Borderline-Persönlichkeitsstörung und die antisoziale Persönlichkeitsstörung nicht behandelt werden. Die Autorinnen weisen darauf hin, dass es aus derselben Reihe zum Thema Borderline einen ausführlichen Band von Martin Bohus gibt. Es bleibt zu hoffen, dass die antisoziale Persönlichkeitsstörung, die im klinischen Alltag durchaus eine Rolle spielt, aber häufig unzureichend versorgt wird, ebenfalls mit einer eigenen Ausgabe bedacht wird.

Fazit: Ein Buch für den psychotherapeutischen Kliniker, das nicht mit einer Vielzahl ätiologischer Modelle oder der Rezitation von Studien ermüdet, sondern mit präzisen und einfach umzusetzenden Anweisungen die Angst vor der therapeutischen Arbeit mit Persönlichkeitsstörungen nehmen soll.

Johannes Hennings, München 